\title{
Medications \& Polypharmacy Influence on Recurrent Fallers in Community: a Systematic Review
}

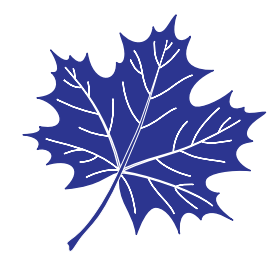

\author{
Yu Ming, MD, $\mathrm{MSc}^{1}$, Aleksandra Zecevic, $\mathrm{PhD}^{2}$ \\ ${ }^{1}$ Graduate Program, Health and Rehabilitation Sciences, Faculty of Health Sciences, Western University, London, ON; \\ ${ }^{2}$ School of Health Studies, Faculty of Health Sciences, Western University, London, ON, Canada
}

https://doi.org/10.5770/cgj.21.268

\begin{abstract}
The purpose of this systematic review is to summarize information about the impact different classes of medications and polypharmacy have on recurrent falls, defined as two or more falls in a 12-month period, in community-dwelling older adults. After adjustment for confounders such as age, gender, weight or depression symptoms, the reviewed studies suggested that older adults who use antidepressants, sedatives or hypnotics and anti-epileptics were more likely to experience recurrent falls than non-users. Polypharmacy (use of four or more prescription medications daily) caused 1.5-2 times higher possibility of recurrent falls in older adults. As a high-risk group, recurrent fallers require meaningful intervention. Medications are believed to be a modifiable risk factor in falls prevention; hence, special consideration should be taken to balance the benefit and harm in initiating, continuing or increasing certain classes of medications in elderly recurrent fallers.
\end{abstract}

Key words: recurrent falls, medications, drugs, polypharmacy, older adults

\section{INTRODUCTION}

Falls can be influential occurrences that cause serious consequences to elderly people physically, psychologically, and economically. Approximately $28-35 \%$ of community-dwelling elderly people, aged 65 years and over, fall each year. ${ }^{(1)}$ About $25 \%$ of elderly people who are 80 years and older fall at least twice annually, ${ }^{(2-4)}$ which imposes substantial cost to the public health system and social services. In Canada, the physical and mental consequences caused by falls lead to a financial cost of about $\$ 2$ billion a year. ${ }^{(5)}$ More alarmingly, the number of fall-related deaths increased by $65 \%$ from 2003 to 2008 . ${ }^{(5)}$

The American Geriatrics Society and British Geriatrics Society have recommended that an investigation on falling two or more times in the past 12 months should be a first screening step in identifying fallers who are in need of intervention. ${ }^{(2)}$ Compared with non-fallers and those who only fall sporadically, recurrent fallers demonstrate less confidence in their ability to perform daily activities, claim more loss of independence, report greater fear of falling, and have an increasing likelihood of admission to a nursing home. . $^{(2,6,7)}$ However, relatively small numbers of studies focused on the mechanism and prevention strategies for recurrent falls. Several studies suggested that some classes of medications, such as antidepressants, sedatives, ${ }^{(8,9)}$ and polypharmacy, ${ }^{(10,11)}$ show significant association with recurrent falls of community-dwelling older adults. Since inappropriate prescription is common among elderly people, ${ }^{(12)}$ reduction or withdrawal of certain classes of medications might be a feasible and effective way to decrease the risk of recurrent falling. Other non-pharmacological treatments, such as acupuncture, psychological or supportive counselling, and exercises, were suggested as a substitute for prescription medications known to lead to recurrent falls among older adults. ${ }^{(13-15)}$

The purpose of this systematic literature review is to summarize findings from studies that explored the association between different classes of medication and polypharmacy and the risk of recurrent falls in people who are 65 years old and over and living in community. The goal of this review is to enhance the medical physicians' and pharmacists' awareness of certain medication classes which may lead to an increased risk of fall recurrences. Even if a total withdrawal of these medications is contradictory in some clinical scenarios, a careful and in-depth consideration on the selection of medications could be beneficial to elderly people who might be in high risk of recurrent falls.

\section{METHODS}

\section{Searching Strategy}

This systematic review consists of articles identified by searching four online databases, namely CINAHL, EMBASE, 
PubMed, and Scopus. Three search themes were combined by the Boolean operator "AND". The first theme, "recurrent falls", combined in title/abstract recurrent fall* or frequent fall* or multiple fall* or repeated fall*. The second theme, "elderly", combined in title/abstract aged, or older adult*, or geriatric*, or elderly, or senior*. The third theme, "medications" combined in medication*, or drug*, or polypharmacy, or Medical Subject Heading $[\mathrm{MeSH}]$ pharmaceutical preparations.

After extracting articles through the initial search strategy, the authors identified the need to include subgroups of medication in the search terms. Papers extracted in the first round of searching were used to identify 18 specific classes of medications associated with recurrent falls. These subclasses of medications were applied during the second round of searching using an 'OR' Boolean operator. They were: ophthalmological*, nasal preparation*, anti-epileptic*, analgesic*, anesthetic*, antibacterial*, beta block*, diuretic*, anti-anemic, laxative*, antithrombotic*, anti-Parkinsonian, antiarrhythmic*, antihypertensive*, sedative*, hypnotic*, anxiolytic*, and antidepressant*. Filters were activated to focus on studies with human subjects, published in English, with no time constraints.

\section{Selection Criteria}

After the literature search was completed and duplicates were removed, the titles and abstracts were reviewed to select the articles that met the following inclusion criteria: (a) used a fall or recurrent falls as an outcome; (b) focused on the association between recurrent falls and medications; and (c) used sample population of older adults age 65 years or older. From the title and abstract information, papers which did not meet the above criteria were excluded. During full-text review of the remaining papers, detailed eligibility criteria were applied. Studies were included if they: 1) provided a definition of recurrent falls, 2) applied adjustment or control for confounders, 3) used ORs (odds ratio) or IRRs (incident rate ratios) together with CIs (confidence intervals) as the outcome measures, and 4) focused on community-dwelling older adults.

Finally, 18 papers were deemed appropriate for inclusion in this literature review. For information that was not provided or was unclear in the selected articles, two authors were contacted by email. One author provided information about the mean age and the percentage of the female participants, ${ }^{(16)}$ and one author answered the question about the length of their follow-up period. ${ }^{(17)}$

\section{Quality and Risk of Bias Assessment of Included Studies}

The 18 articles included in this review were all observational studies. Observational studies are ideally designed for the assessment of low-incidence events such as falls among the general population. Furthermore, the selection of participants in observational studies is a better indication of daily medical practice. However, assessment of quality and susceptibility to bias for observational studies remains a methodological problem. ${ }^{(18)}$ Hence, we used Strengthening the Reporting of Observational Studies in Epidemiology (STROBE) ${ }^{(19)}$ checklist, to capture the key study design features. The appraisal was completed by two raters (YM and UA) for all 18 observational studies included in this review. The two raters reached consensus by discussion (Table 1).

Two raters also independently evaluated the risk of bias in the included studies using the refined version of Quality in Prognosis Factors (QUIPS) tool. ${ }^{(20)}$ The risk was based on six domains: study participation, study attrition, prognostic factors measurement, outcome measurement, study confounding, statistical analysis and reporting. Each domain contained three to seven components, and these components were used to render low, moderate or high risk of bias. The risk evaluation is summarized in Table 2.

\section{Analysis and Synthesis of the Literature}

The location of the study, sample size, percentage of female participants, mean age, recurrent fall definition, study design, characteristics of comparison group, and confounders or regression analysis models applied, were extracted from each article and tabulated. The ORs or IRRs, together with CIs for each medication, were extracted and the medications were grouped according to their clinical application. Two studies, conducted by Rossat et al. in 2010 and $2011^{(21,22)}$ applied IRR as the outcome measures. The ORs for the same class of medications were compared across studies to provide a clear picture of the extent to which each medication class and polypharmacy may influence elderly recurrent fallers. PRISMA (Preferred Reporting Items for Systematic Reviews and Meta-Analyses) was used to report findings of this systematic review. ${ }^{(23)}$ To confirm the results of this review, sensitivity analysis using meta-analysis was performed for well-designed, prospective studies using Revman 5.3.

\section{RESULTS}

The CINAHL, EMBASE, PubMed, and Scopus search produced 68, 329, 198, and 248 citations, respectively. Duplicates $(\mathrm{N}=458)$ were removed and after review of titles and abstracts, 394 papers were removed because they did not meet the inclusion criteria. Sixty-four full-text research papers were reviewed and 46 were eliminated for not meeting the detailed eligibility criteria. The remaining 18 papers, which met all eligibility criteria, were included in this literature review (Figure 1).

The characteristics of participants and study designs were summarized in Table 3. Eight studies were prospective cohorts, one was a retrospective cohort, and the remaining eleven were cross-sectional studies. Nine studies selected non-fallers as a comparison to calculate the OR of recurrent fallers, two studies used single fallers (one fall during the previous 12 
MING: DRUGS' INFLUENCE ON RECURRENT FALLERS

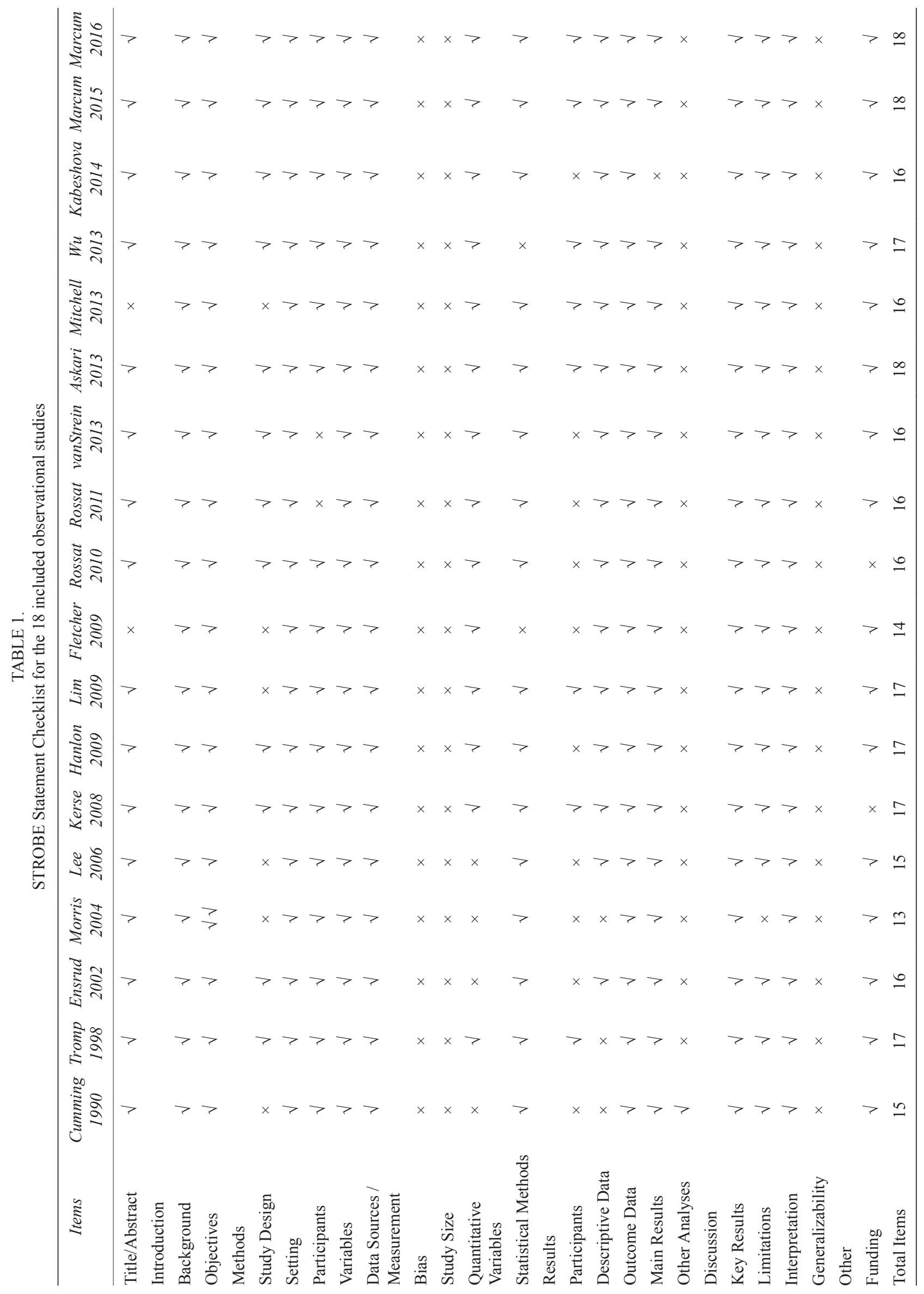

CANADIAN GERIATRICS JOURNAL, VOLUME 21, ISSUE 1, MARCH 2018 


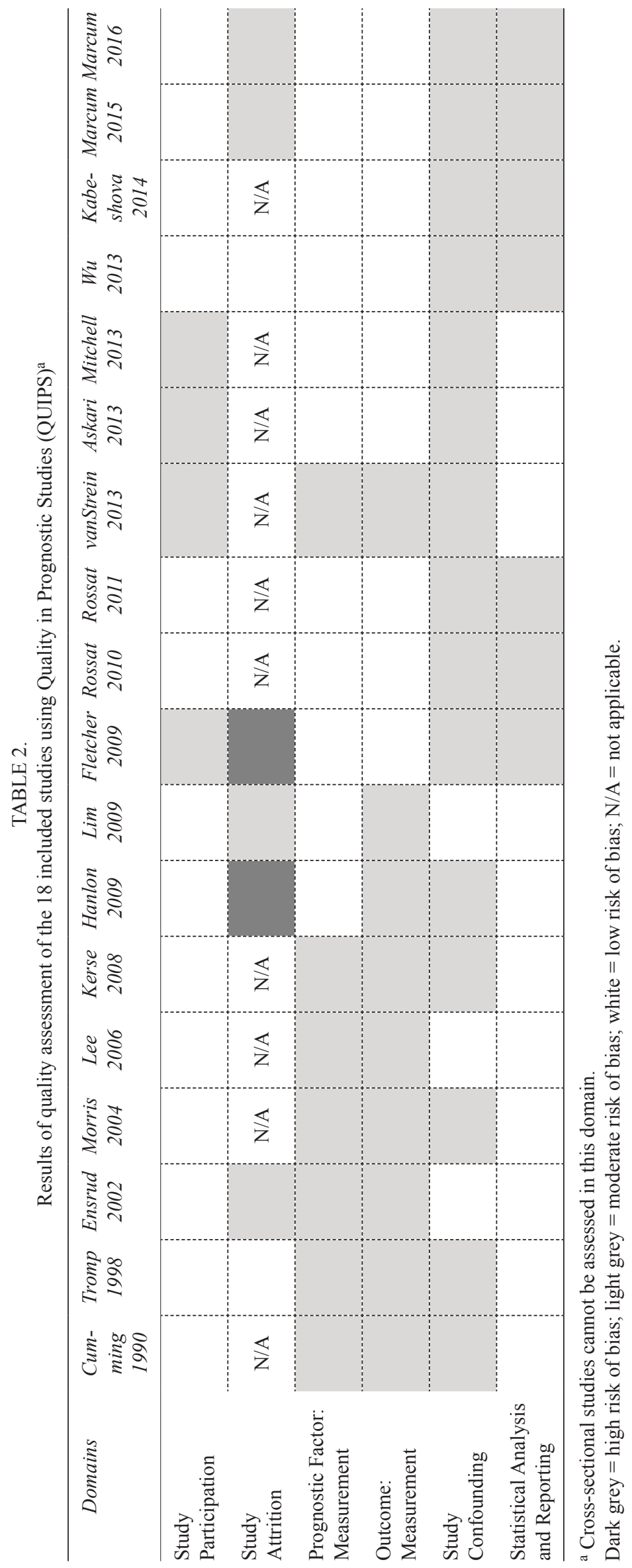




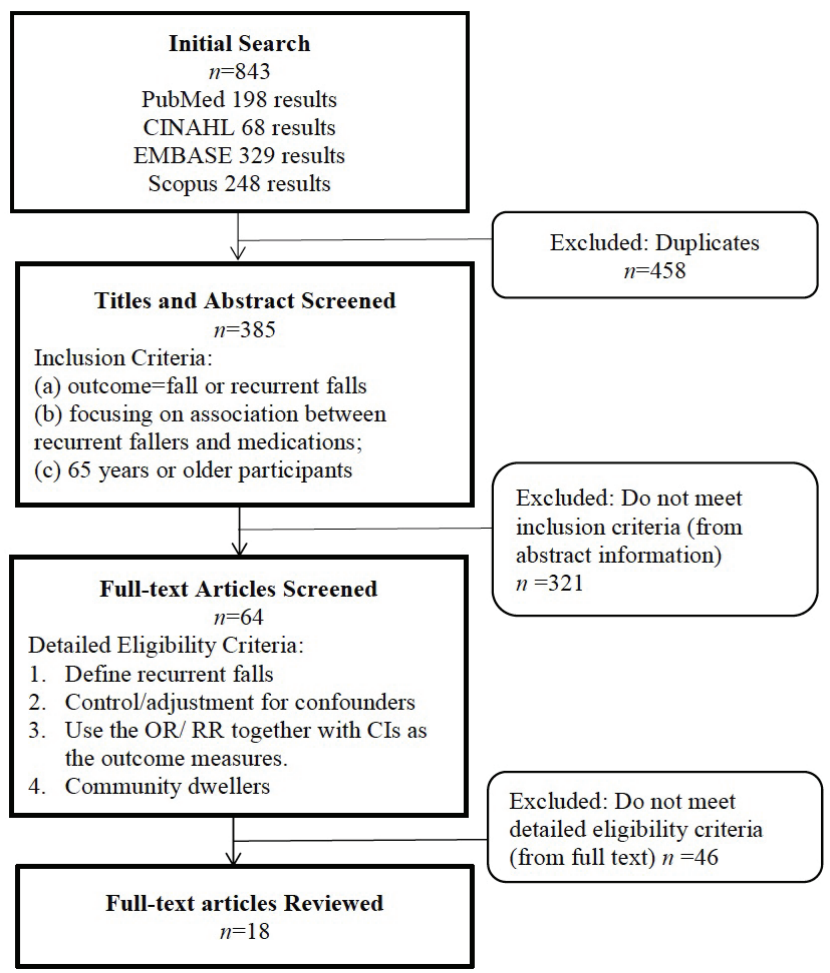

FIGURE 1. Flow chart of searching and selection of included papers

months) for comparison, and the other seven studies compared recurrent fallers with older adults who experienced no falls or one fall in the past 12 months. Participants in 17 studies were asked to recall their falls in the past 12 months, while Ensrud et al. ${ }^{(24)}$ called their participants for fall information every 4 months, over the 12-month follow-up period. All studies used interviews with the participants to ascertain the medications used by participants at the time of the study.

As is shown in Table 3, 16 out of 18 included studies adopted similar criteria of recurrent fallers, which are defined as people who experienced two or more falls in a 12-month period. Rossat et al..$^{(21)}$ and van Strien et al.$^{(9)}$ defined recurrent fallers as people who fall more than twice in a 12-month period.

Table 4 shows a summary of the ORs or RRs (together with CIs) of medications that were evaluated in included studies. This literature review classified the medications based on the second level of Anatomical Therapeutic Chemical (ATC) classification and their clinical application. The classes were: 1. Psychotropic medications, such as "antidepressants (N06A)", "sedatives/hypnotics (N05B)", "anti-epileptics (N03A)", "Narcotics/ analgesics (N02A)" "antipsychotics (N05A)", and "anti-Parkinson drugs (N04A)"; 2. Cardiovascular disease related medications; 3 . Other medications, such as "laxatives (A06A)", "anti-diabetic drugs (A10B)", "nasal preparations (R01A)", and "ophthalmologicals (S01)"; and 4. Polypharmacy, defined by four of the included studies as using of at least four prescription medications daily.

\section{Psychotropic Medications}

Psychotropic medications refer to chemicals that affect the central nervous system (CNS), which can alter the psychological processes such as mood, emotion, perception, and behaviour. ${ }^{(25)}$ The drugs were classified into subgroups according to their clinical application, for a clear understanding of their impact on recurrent falls. The following is a summary of their impact, drawn from the conclusions from the included studies.

\section{Antidepressants}

Six studies investigated the association between antidepressants and recurrent falls and five of them applied adjustment on age, sex or other health indicators. All the studies ${ }^{(8,9,17,24,26,27)}$ found that the use of antidepressants was associated with recurrent falls. Despite of the fact that they used different control groups for comparison, the crude ORs ranged from 1.97 to 3.33 and the adjusted ORs ranged from 1.46 to 3.09 , which indicated that older adults who took antidepressants were 1.5 to 3 times more likely to experience multiple falls, compared to those who did not take them. Five studies with adjusted $\mathrm{ORs}^{(8,9,17,24,27)}$ provided the confidence intervals' lower limits above the null value $(\mathrm{OR}=1.0)$, which is an indication that the results were of statistical importance. Initially, a meta-analysis of these five studies showed that the heterogeneity was high $\left(\mathrm{I}^{2}=100 \%\right)$. Authors suspected that this was because three studies used non-fallers as a control group ${ }^{(17,24,27)}$ one used single fallers, ${ }^{(8)}$ and another used non-recurrent fallers ${ }^{(9)}$ as comparisons. Next, a meta-analysis was performed using three studies that used only non-fallers as control groups, but the $\mathrm{I}^{2}$ was $85 \%$. Therefore, we performed anther analysis which only contained prospective cohort studies. ${ }^{(24,27)}$ Figure 2 shows that a pooled OR of 2.37 (95\% CI 1.95-2.88) was obtained through a meta-analysis of these two prospective studies, with $\mathrm{I}^{2}=0 \% p=0.82$.

Of all the antidepressants that are usually prescribed to Canadian seniors, selective serotonin reuptake inhibitors (SSRIs) and tricyclic antidepressants (TCAs) were the most frequently prescribed. ${ }^{(28)}$ Marcum et al. ${ }^{(27)}$ found that both SSRIs and TCAs were related to recurrent falls in older adults (aOR 1.62 and 1.27, respectively). Ensrud et al. ${ }^{(24)}$ verified that SSRI users were 3.45 times more likely than non-users to fall frequently, with $95 \% \mathrm{CI}=1.89-6.30$. The study of Kerse et al. ${ }^{(17)}$ also confirmed this with an adjusted OR of 1.66, and 95\% CI $=1.36-2.02$. Cumming et al $^{(26)}$ found that TCAs were 1.7 times more likely to cause recurrent falls than 'no falls' or a 'single fall'.

\section{Sedatives and Hypnotics}

Recent studies conducted by Askari et al., ${ }^{(8)}$ van Strien et al., ${ }^{(9)}$ and $\mathrm{Wu}$ et al. ${ }^{(10)}$ showed that elderly people who took sedatives and hypnotics were 1.8 to 4.5 times more likely to experience recurrent falls. Even after adjustment for 


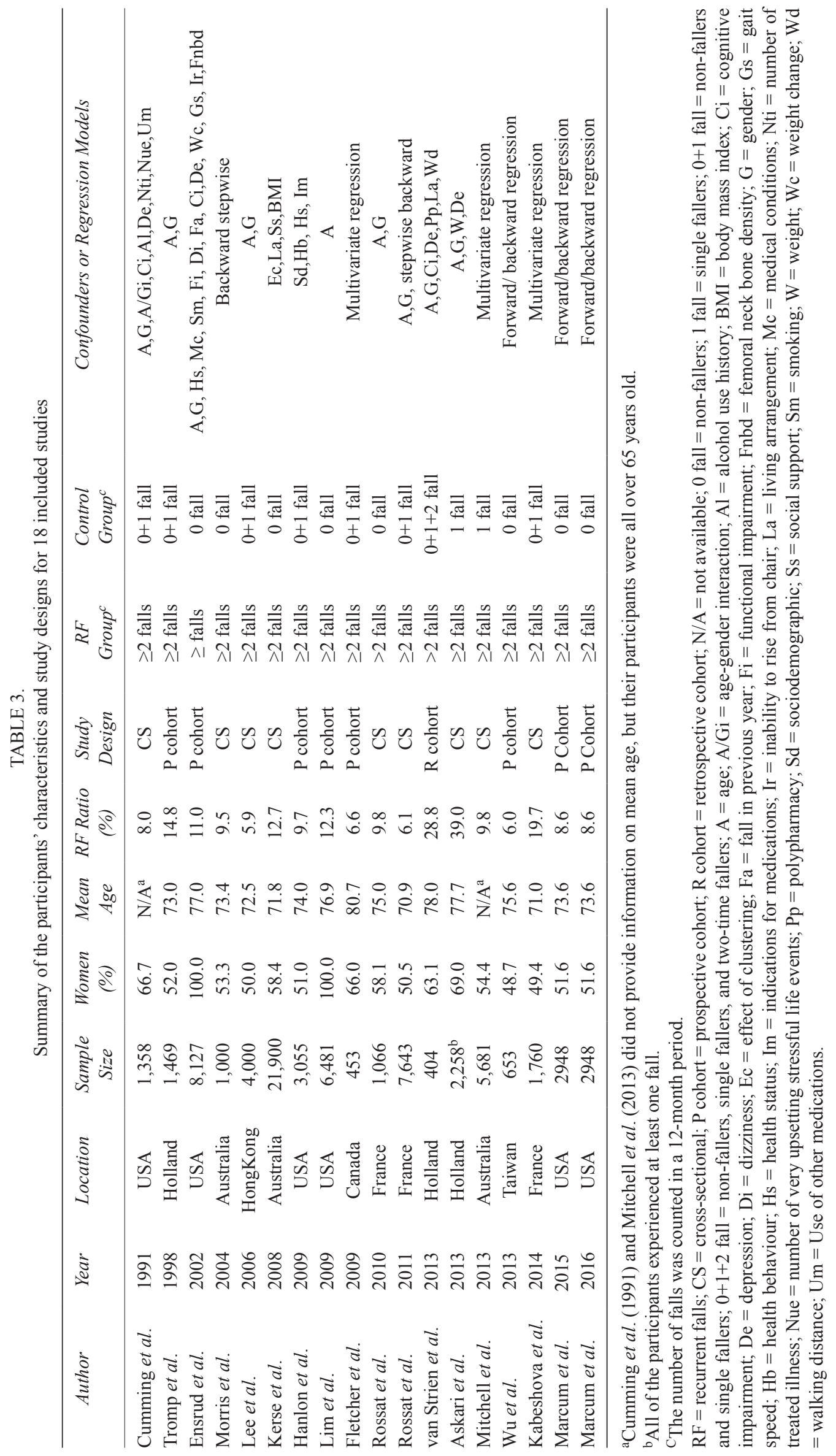



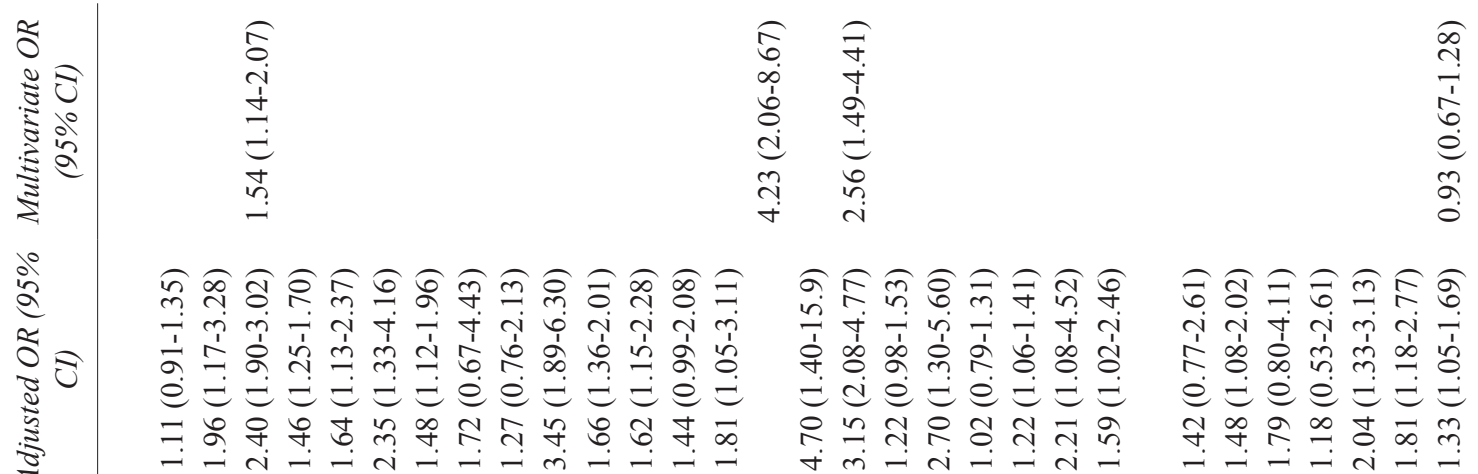

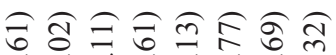

iิ

o.

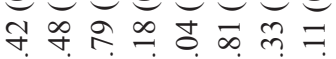

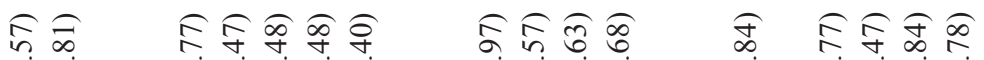

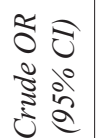

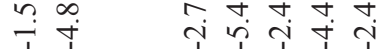

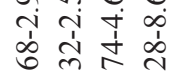

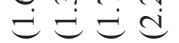

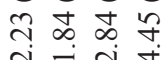

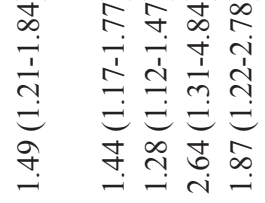

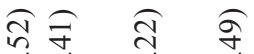
○

ते :

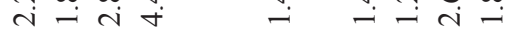

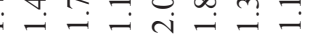

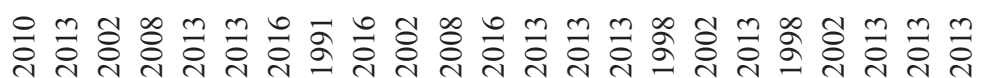

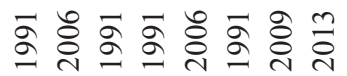
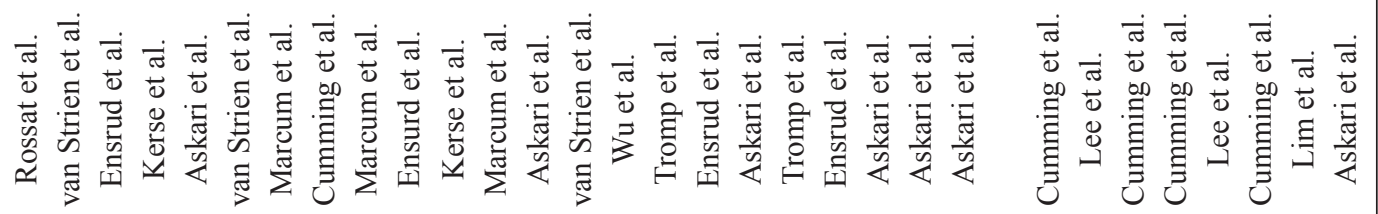

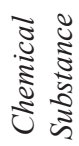

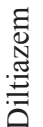

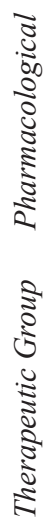

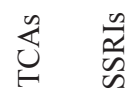

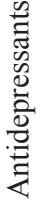

00
0
0
0
0
0
0
0
0
0
0
0
0
0
0

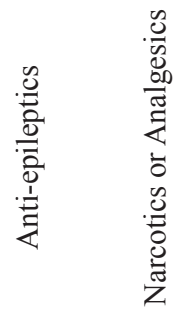

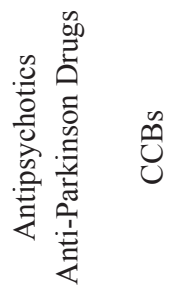

苞

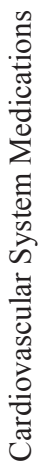




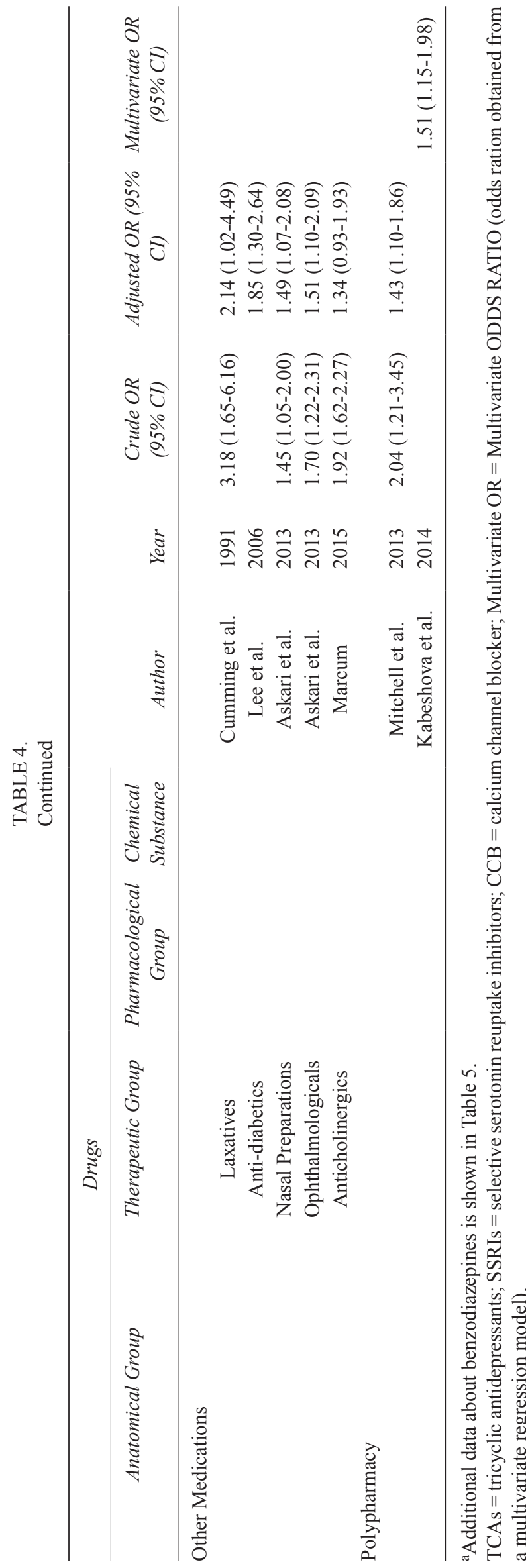

confounders such as age and gender, or running a forward entry or backward elimination regression model, the risk of falling repeatedly after taking sedatives or hypnotics was 1.4 to 4.2 times higher than for older adults who were not taking these medications. The influence of benzodiazepines (BDZs), one of the most commonly used hypnotics in the general population ${ }^{(29)}$ on elderly recurrent fallers, is summarized in Table 5. BDZs with half-life more than 24 hours, 12 to 24 hours and less than 12 hours were categorized as long-acting, intermediate-acting, and short-acting, respectively. Cumming et al. ${ }^{(26)}$ and Ensrud et al. ${ }^{(24)}$ reported respectively that users of long-acting BDZs were 2.18 and 1.56 times more likely to fall repeatedly, compared to those who did not take long-acting BDZs. Although none of included studies explicitly focused on immediate-acting BDZs, Rossat et al. ${ }^{(22)}$ reported that two specific medications, bromazepam and clobazam, which belong to immediate-acting BDZs, were related to recurrent falls. van Strien and colleagues ${ }^{(9)}$ demonstrated that older adults who took short-acting benzodiazepines were 1.94 times more likely to experience repeated falls, even after considering the influence of factors like age, gender, cognitive impairment, depression, polypharmacy, living situation, and walking distance.

\section{Anti-epileptics}

Adjusted or multivariate ORs of three studies ${ }^{(8,24,30)}$ have shown that anti-epileptics may increase the risk of falling frequently in community-dwelling older adults, compared with the risk of no fall or falling just once. Ensrud et al. (24) concluded that users of anti-epileptics were 3.15 times more likely to experience repeated falls ( $\mathrm{OR}=3.1595 \% \mathrm{CI}=2.08$ 4.77). Another study conducted by Tromp et al. ${ }^{(30)}$ showed that elderly people who took anti-epileptics had 4.7 times the risk of falling recurrently, in comparison with experiencing no fall or single fall. Askari et al. ${ }^{(8)}$ found a moderate relationship (OR=1.22 95\% CI=0.98-1.53) between anti-epileptics and recurrent falls.

\section{Narcotics or Analgesics}

The influence of narcotics or analgesics was not clearly established in the included studies. Tromp et al. ${ }^{(30)}$ concluded that the use of analgesics was strongly related to recurrent falls, with an adjusted OR value of 2.7 and $95 \%$ CI of 1.30-5.60. Cumming et al. ${ }^{(26)}$ reported that taking no confounders into consideration, analgesic users were 1.56 times more likely to fall recurrently, but after adjustment for age, age-gender interaction, and depression, they failed to find a meaningful relationship between analgesics and recurrent falls. Similarly, Ensrud et al. ${ }^{(24)}$ revealed that, after controlling for age, health status, functional impairment, dizziness, cognitive function, and depression as the confounders simultaneously, there was no difference in the risk of frequent falls in women whether they took narcotics or not. Askari et al. ${ }^{(8)}$ showed that older adults who took analgesics were 1.22 times more likely to fall 


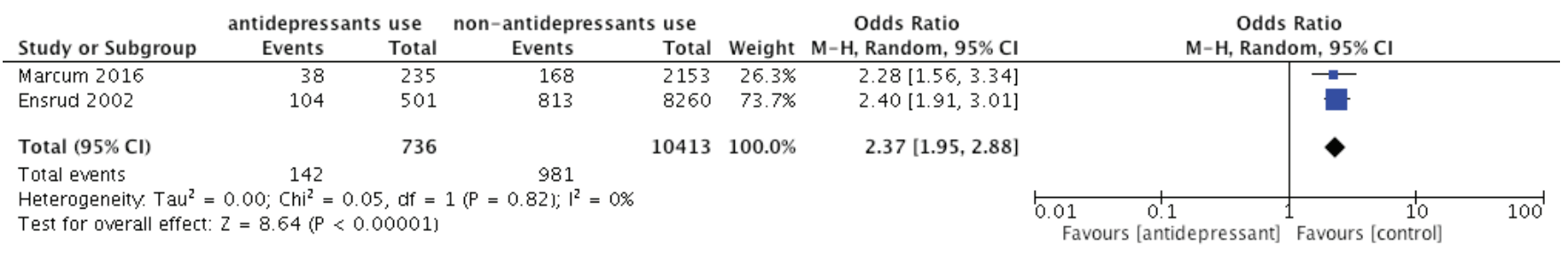

FIGURE 2. Use of antidepressants and recurrent falls: meta-analysis of two prospective cohort studies

repeatedly than those who did not take analgesics, but they admitted that this cannot be regarded as a strong association between analgesics and recurrent falls. They also investigated antipsychotics and anti-Parkinsonian drugs and found that elderly people who took these two classes of drugs were 2.21 and 1.59 times, respectively, more likely to experience multiple falls than those who did not take them. ${ }^{(8)}$

\section{Cardiovascular System Medications}

Cumming et al. ${ }^{(26)}$ found that Diltiazem, a calcium antagonist, was associated with recurrent falls, but after adjustment for variables like age, sex, depression, cognitive impairment, the confidence intervals of the OR crossed the 1.0 borderline, indicating non-significant relationship. Users of vasodilators, such as nitrate, were found to be two times more likely to experience recurrent falls. ${ }^{(25,31)}$ But when Cumming and colleagues ${ }^{(26)}$ adjusted ORs for confounding factors, they were not able to find meaningful association. Diuretics are a group of medications which are used extensively among the older adults for cardiovascular and renal diseases, but their relationships with recurrent falls has yet to be confirmed. Cumming et $a l .{ }^{(26)}$ reported that there was a 1.8 times increased likelihood of recurrent falls in diuretics users, while Askari et al. ${ }^{\left({ }^{(8)}\right.}$ and Lim et al. ${ }^{(32)}$ did not find association between diuretics and recurrent falls.

\section{Other Medications}

Cumming et al. ${ }^{(26)}$ reported that laxatives may cause 2.1 times more risks in falling repeatedly. Lee et al. ${ }^{(31)}$ showed that anti-diabetics had a moderate association with recurrent falls after adjustment for eye diseases or heart diseases. Askari et $a l .{ }^{(8)}$ focused on the association between nasal preparations and ophthalmologicals and recurrent falls, and found that after adjustment for age, sex, weight, and depression, older adults who used these two classes of drugs were nearly 1.5 times more likely to fall repeatedly than those who did not use them. Marcum et al. ${ }^{(33)}$ recently found that anticholinergic medication use in older adults may cause 1.34 times likelihood of recurrent falls.

\section{Polypharmacy}

A number of researchers who focused on recurrent falls defined polypharmacy as taking four or more prescription medications daily. ${ }^{(10,30,34)}$ They found that polypharmacy was positively related to recurrent falls. A multiple logistic regression model reported by Kabeshova et $_{\text {al. }}{ }^{(34)}$ revealed that polypharmacy was associated with recurrent falls $(\mathrm{MOR}=1.51$, $95 \% \mathrm{CI}=1.15-1.98)$. Mitchell et al. ${ }^{(16)}$ found similar results: $\mathrm{OR}=1.43,95 \mathrm{CI}=1.10-1.86$ after adjustment for age and gender. Morris et al., ${ }^{(35)}$ Tromp et al., ${ }^{(30)}$ and $\mathrm{Wu}$ et al. ${ }^{(10)}$ showed that compared with a single fall, polypharmacy was a significant predictor of multiple falls before adjustment for other confounding factors. But when performing multivariate regression models, they failed to report a significant influence of polypharmacy. Another concerning fact reported by Hanlon et al. ${ }^{(36)}$ was that, when more psychotropic drugs were in the regimen, the patients were more likely to fall repeatedly. Older adults taking two or more psychotropic agents were almost two times $(\mathrm{aOR}=1.95,95 \% \mathrm{CI}=1.35-2.81)$ more likely to fall recurrently, compared to those who did not take any psychotic agents. ${ }^{(36)}$ Fletcher et al. ${ }^{(37)}$ concluded that every prescription medication added to the daily regimen increased the risk of falling recurrently 1.21 times $(95 \%$ CI $1.02-1.43)$.

\section{DISCUSSION}

Since the early 90's, several systematic reviews and metaanalyses concerning the relationship between medications and falls have been published. ${ }^{(38-41)}$ That being said, systematic reviews and meta-analyses on the medications which are correlated to recurrent fallers are scarce. This systematic literature review identified 18 studies that reported the association between diverse medication classes and recurrent falls, and provided a summary of their results.

Sixteen out of 18 included studies adopted two or more falls in a 12-month period as the definition of recurrent falls. Fifteen studies provided information on recurrent falls incidence rate or relative data for the authors to calculate the recurrent falls incidence rate in population of seniors (Table $3)$. The mean recurrent fall incidence rate of the 15 studies was $10.16 \pm 4.02 \%$, which compares well with other studies reporting recurrent falls rates. ${ }^{(42,43,44)}$

This systematic review investigated the influence of psychotropic drugs, cardiovascular drugs, other drugs such as laxatives, anti-diabetic agents, and nasal preparations on recurrent falls of older adults who live in the community. Psychotropic drugs are usually administered to community-dwelling elderly people to help them cope with psychological problems such as anxiety, depression, and insomnia. Ramage-Morin ${ }^{(45)}$ 


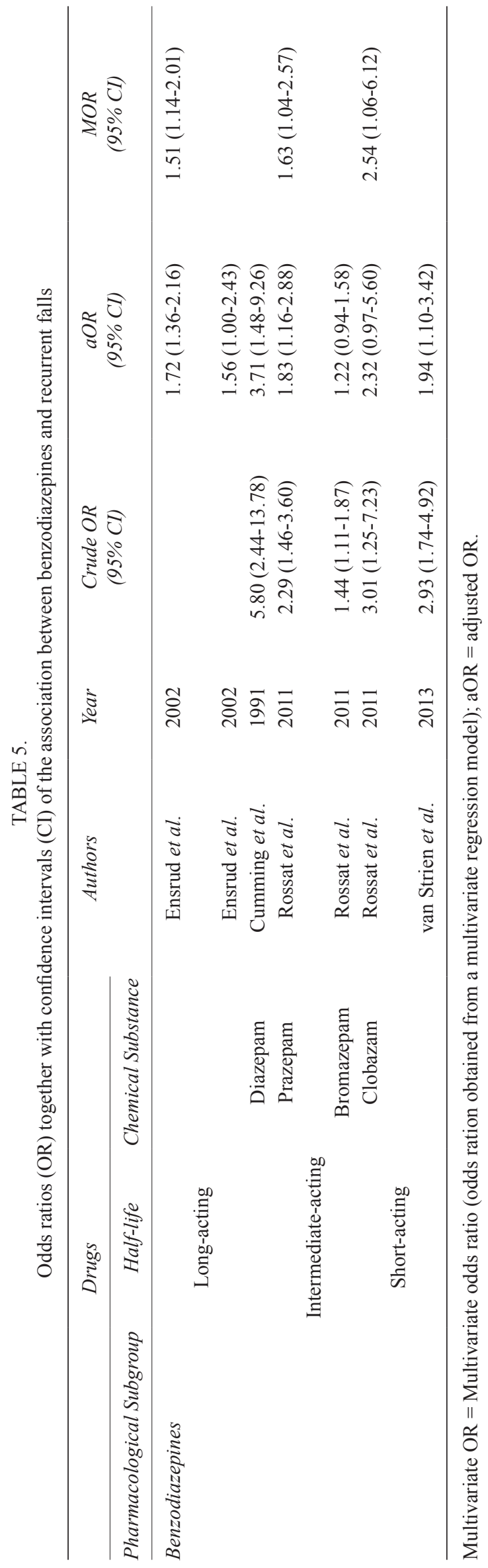

investigated the medications that Canadian seniors often used and reported that $78 \%$ institutionalized and $37 \%$ communitydwelling older adults took medications for nervous system disorders. All studies included in this systematic review that focused on association between antidepressants, sedatives or hypnotics, anti-epileptics and recurrent falls verified that these classes of medications could lead to increasing risk of recurrent falls. Even after adjustment for confounders, the relationship between these psychotropic medications and recurrent falls remained significant. These findings showed consistency in the direction of the effect, and are biologically and pharmacologically plausible. These results are consistent with findings of meta-analyses which focused on the relationship between psychotropic agents and falls. ${ }^{(38,39)}$

Antidepressants are commonly used in older adults to treat depression, anxiety disorders, and chronic neuropathic pain. Antidepressants are believed to increase the risk of falls through mechanisms such as causing sedation or postural hypotension, impairing balance, delaying reaction times, and deranging sleeping structure resulting in daytime drowsiness and nocturia. The sensitivity analysis confirmed the conclusion of this review that antidepressants use may double the risk of falling recurrently.

The use of sedatives or hypnotics is frequent in elderly people with reported prevalence rate of $16-28.7 \%$. $^{(46,47)}$ The indications for use of BZDs are anxiety, adjustment disorders and insomnias, but they can cause impaired attention and gait and balance disorders. The effects of benzodiazepines on recurrent falls have been investigated in four of studies included in this literature review. Overall, older adults who took BDZs were exposed to a 1.2 to 3.7 times greater risk of falling repeatedly.

Although a clear and widely accepted definition of polypharmacy does not exist, it is a well-established that polypharmacy is related to the onset of unfavourable health outcomes. ${ }^{(48)}$ It is reported that, although elderly Americans comprised 13\% of the population, they consume one-third of the prescription medications every year. ${ }^{(49)}$ Falls are one of the serious health problems that may be ascribed to adverse drug events of polypharmacy. Findings of this review show that polypharmacy might increase the possibility of falling repeatedly $1.5-2$ times.

This review has several strengths. First, all the included studies had large sample size, thus increasing the chance of identifying a significant difference while avoiding selection bias. Second, 16 out of 18 studies adopted the same criterion for recurrent falls, which ensured a homogeneous population and made the results more comparable. Third, studies which did not adjust for confounders, such as age and gender, were not included in this review because they only considered the effect of one independent variable. However, falls are caused by complex interaction between the internal and external risk factors, which cannot be explained by a single variable. Studies providing adjustment for confounders such as age, sex, depression, and other health indicators were of higher methodological quality and their results were more convincible in real clinical scenarios. Finally, the results of sensitivity analysis were 
consistent with the overall finding of this review. A metaanalysis pooled OR can be regarded as a strong evidence of the association between antidepressants and recurrent falls.

Several limitations of this review also need to be considered. At first, setting a language limitation to English might have acted as a barrier to the inclusion of more studies conducted in Europe and Asia. Second, the retrospective or cross-sectional designs posed a threat to the reliability of 10 included studies. However, studies on recurrent falls are mainly cohort or cross-sectional studies because the incidence of recurrent falls is low and it would take decades to collect a satisfying number of events for randomized trials. Third, falls and medications were ascertained by interviews. These selfreported results carry a risk of recall bias and under-reporting of recurrent falls or use of certain classes of medications. Fourth, as is common in this type of research, it is possible that confounding by indications and residual unmeasured confounding factors, not considered in the included studies, influenced the results of this review.

\section{CONCLUSIONS}

After adjustment for confounders such as age, gender, weight or depression symptoms, the reviewed studies suggested that community-dwelling older adults who used antidepressants, sedatives or hypnotics (especially benzodiazepines) and anti-epileptics were more likely to experience recurrent falls than older adults not using these medication classes. Polypharmacy was moderately related (1.5-2 times) to repeated falls of elderly people. The influence of other medications, such as agents for cardiovascular diseases, laxatives, and anti-diabetics, remains inconclusive. As recurrent fallers are in greater need for falls prevention and medications are believed to be modifiable risk factors in falls prevention programs, well-designed, large sample size randomized control trials are needed to explore a potential for reduction of fall risk by changing prescribing practices, and to assist clinicians and pharmacists to provide more individualized pharmacotherapy to those patients who are at higher risk of falling recurrently.

\section{ACKNOWLEDGEMENTS}

The authors thank Udani Atukoralalage and Alma Nikolic for their methodological support and advice.

\section{CONFLICT OF INTEREST DISCLOSURES}

The authors declare that no conflicts of interest exist.

\section{REFERENCES}

1. World Health Organization. WHO Global Report on Falls Prevention in Older Age. Geneva: WHO; 2008. Available from: http:// www.who.int/ageing/publications/Falls_prevention7March. pdf?ua $=1$
2. Panel on Prevention of Falls in Older Persons, American Geriatrics Society and British Geriatrics Society. Summary of the updated American Geriatrics Society/British Geriatrics Society clinical practice guideline for prevention of falls in older persons. J Am Geriatr Soc. 2011;59(1):148-57.

3. Rubenstein LZ. Falls in older people: epidemiology, risk factors and strategies for prevention. Age Ageing. 2006;35(suppl 2):ii37-ii41.

4. Tinetti ME. Preventing falls in elderly persons. $N$ Engl J Med. 2003;348(1):42-49.

5. Public Health Agency of Canada. Seniors' Falls in Canada: Second Report. Ottawa: The Agency; 2014. Retrieved from http:/www.phac-aspc.gc.ca/seniors-aines/publications/ public/injury-blessure/seniors_falls-chutes_aines/assets/pdf/ seniors_falls-chutes_aines-eng.pdf

6. Kelsey JL, Procter-Gray E, Berry SD, et al. Re-evaluating the implications of recurrent falls in older adults: location changes the inference. J Am Geriatr Soc. 2012;60(3):517-24.

7. Tinetti ME, Doucette J, Claus E, et al. Risk factors for serious injury during falls by older persons in the community. $\mathrm{J} \mathrm{Am}$ Geriatr Soc. 1995;43(11):1214-21.

8. Askari M, Eslami S, Scheffer AC, et al. Different riskincreasing drugs in recurrent versus single fallers: are recurrent fallers a distinct population? Drugs and Aging. 2013;30(10):845-51.

9. van Strien AM, Koek HL, van Marum RJ, et al. Psychotropic medications, including short acting benzodiazepines, strongly increase the frequency of falls in elderly. Maturitas. 2013;74(4):357-62.

10. Wu TY, Chie WC, Yang RS, et al. Risk factors for single and recurrent falls: a prospective study of falls in community dwelling seniors without cognitive impairment. Prev Med. 2013;57(5):511-17.

11. Freeland $\mathrm{KN}$, Thompson AN, Zhao YM, et al. Medication Use and Associated Risk of Falling in a Geriatric Outpatient Population. Ann Pharmacother. 2012;46(9):1188-91.

12. Campanelli CM. American Geriatrics Society Updated Beers Criteria for potentially inappropriate medication use in older adults. J Am Geriatr Soc. 2012;60(4):616-31.

13. Gao XY, Xu CX, Chen XW, et al. Clinical research on insomnia treated by acupuncture at back-shu points. World J AcupunctureMoxibustion. 2014;24(2):1264-66.

14. Cuijpers P, Karyotaki E, Pot AM, et al. Managing depression in older age: psychological interventions. Maturitas. 2014;79(2):160-69.

15. Schuch FB, Vancampfort D, Rosenbaum S, et al. Exercise for depression in older adults: a meta-analysis of randomized controlled trials adjusting for publication bias. Rev Brasil Psiquiatr. 2016;38(3):247-54

16. Mitchell RJ, Watson WL, Milat A, et al. Health and lifestyle risk factors for falls in a large population-based sample of older people in Australia. J Safety Res. 2013;45:7-13.

17. Kerse N, Flicker L, Pfaff JJ, et al. Falls, depression and antidepressants in later life: a large primary care appraisal. PLoS ONE. 2008;3(6):e2423. 
18. Sanderson S, Tatt ID, Higgins JP. Tools for assessing quality and susceptibility to bias in observational studies in epidemiology: a systematic review and annotated bibliography. Int J Epidemiol. 2007;36(3):666-76.

19. Von Elm E, Altman DG, Egger M, et al. The Strengthening the Reporting of Observational Studies in Epidemiology (STROBE) statement: guidelines for reporting observational studies. Prev med. 2007;45(4):247-51.

20. Hayden JA, van der Wíndt DA, Cartwright JL, et al. Assessing bias in studies of prognostic factors. Ann Intern Med. 2013;158(4):280-86.

21. Rossat A, Fantino B, Nitenberg C, et al. Risk factors for falling in community-dwelling older adults: which of them are associated with the recurrence of falls? J Nutr Health Aging. 2010;14(9):787-91.

22. Rossat A, Fantino B, Bongue B, et al. Association between benzodiazepines and recurrent falls: a cross-sectional elderly population-based study. J Nutr Health Aging. 2011;15(1):72-77.

23. Moher D, Liberati A, Tetzlaff J, et al. Preferred reporting items for systematic reviews and meta-analyses: the PRISMA statement. Ann of Intern Med. 2009;151(4):264-69.

24. Ensrud KE, Blackwell TL, Mangione CM, et al. Central nervous system-active medications and risk for falls in older women. $J$ Am Geriatr Soc. 2002;50(10):1629-37.

25. Gorman JM. The Essential Guide to Psychiatric Drugs, 4th edition. New York: St. Martin's Griffin; 2007.

26. Cumming RG, Miller JP, Kelsey JL, et al. Medications and multiple falls in elderly people: the St Louis OASIS study. Age Ageing. 1991;20(6):455-61.

27. Marcum ZA, Perera S, Thorpe JM, et al. Antidepressant use and recurrent falls in community-dwelling older adults: findings from the Health ABC Study. Ann Pharmacother. 2016;50(7):525-33.

28. Wiese BS. Geriatric depression: the use of antidepressants in the elderly. BCMJ. 2011;53(7):341-47.

29. Morlock RJ, Tan M, Mitchell DY. Patient characteristics and patterns of drug use for sleep complaints in the United States: analysis of National Ambulatory Medical Survey data, 1997-2002. Clin Ther. 2006;28(7):1044-53.

30. Tromp AM, Smit JH, Deeg DJ, et al. Predictors for falls and fractures in the Longitudinal Aging Study Amsterdam. J Bone Miner Res. 1998;13(12):1932-39.

31. Lee, JSW, Kwok T, Leung PC, et al. Medical illnesses are more important than medications as risk factors of falls in older community dwellers? A cross-sectional study. Age Ageing. 2006;35(3):246-51.

32. Lim LS, Fink HA, Blackwell T, et al. Loop diuretic use and rates of hip bone loss and risk of falls and fractures in older women. J Am Geriatr Soc. 2009;57(5):855-62.

33. Marcum ZA, Perera S, Thorpe JM, et al. Anticholinergic use and recurrent falls in community-dwelling older adults: findings from the Health ABC Study. Ann Pharmacother. 2015;49(11):1214-21.

34. Kaveshova A, Annweiler C, Fantino B, et al. A regression tree for identifying combinations of fall risk factors associated to recurrent falling: a cross-sectional elderly population-based study. Aging Clin Exp Res. 2014;26(3):331-36.

35. Morris M, Osborne D, Hill K, et al. Predisposing factors for occasional and multiple falls in older Australians who live at home. Aust J Physiother. 2004;50(3):153-59.

36. Hanlon JT, Boudreau RM, Roumani YF, et al. Number and dosage of central nervous system medications on recurrent falls in community elders: the Health, Aging and Body Composition study. J Gerontol A Biol Sci Med Sci. 2009;64(4):492-98.

37. Fletcher PC, Berg K, Dalby DM, et al. Risk factors for falling among community-based seniors. J Patient Saf. 2009;5(2):61-66.

38. Woolcott JC, Richardson KJ, Wiens MO, et al. Meta-analysis of the impact of 9 medication classes on falls in elderly person. Arch Intern Med. 2009;169(21):1952-60.

39. Leipzig RM, Cumming RG, Tinetti ME. Drugs and falls in older people: a systematic review and meta-analysis: I. Psychotropic drugs. J Am Geriatr Soc. 1999;47(1):30-39.

40. Leipzig RM, Cumming RG, Tinetti ME. Drugs and falls in older people: a systematic review and meta-analysis: II. Cardiac and analgesic drugs. J Am Geriatr Soc. 1999;47(1):40-50.

41. Deandrea S, Lucenteforte $\mathrm{E}$, Bravi F, et al. Risk factors for falls in community-dwelling older people: a systematic review and meta-analysis. Epidemiology. 2010;21(5):658-68.

42. Pluijm SM, Smit JH, Tromp EA, et al. A risk profile for identifying community-dwelling elderly with a high risk of recurrent falling: results of a 3-year prospective study. Osteoporos Int. 2006;17(3):417-25.

43. Yoo IY. Recurrent falls among community-dwelling older Koreans: prevalence and multivariate risk factors. $J$ Gerontol Nurs. 2011;37(9):28-40.

44. Stalenhoef PA, Diederiks JP, Knottnerus JA, et al. A risk model for the prediction of recurrent falls in communitydwelling elderly: a prospective cohort study. J Clin Epidemiol. 2002;55(11):1088-94.

45. Ramage-Morin PL. Medication use among senior Canadians. Health Rep. 2009;20(1):37-44.

46. Egan M, Moride Y, Wolfson C, et al. Long-term continuous use of benzodiazepines by older adults in Quebec: prevalence, incidence and risk factors. J Am Geriatr Soc. 2000;48(7):811-16.

47. Lechevallier N, Fourrier A, Berr C. [Benzodiazepines use in the elderly: the EVA study] [in French]. Rev Epidemiol Sante Publique. 2003;51(3):317-26.

48. Viktil KK, Blix HS, Moger TA, et al. Polypharmacy as commonly defined is an indicator of limited value in the assessment of drug-related problems. Brit J Clin Pharmacol. 2007;63(2):187-95.

49. Bushardt RL, Massey EB, Simpson TW, et al. Polypharmacy: misleading, but manageable. Clin Interv Aging. 2008;3(2): 383-89.

Correspondence to: Yu Ming, MD, MSc. Graduate Program, Health and Rehabilitation Sciences, Faculty of Health Sciences, Western University, Elborn College, Room 1014, London, ON, Canada, N6G 1H1

E-mail: yming5@uwo.ca 